\title{
Estrategias de comunicación interactivas en los sitios web de marcas de alimentación dirigidas al público infantil
}

\author{
María Henar AlONSO MOSQUERA \\ María SÁNCHEZ MARTÍNEZ ${ }^{1}$ \\ Universidad San Pablo CEU
}

\begin{abstract}
RESUMEN:
El presente trabajo forma parte de una investigación más amplia acerca de la publicidad enfocada a los niños y adolescentes en entornos interactivos.

En la actualidad y debido a la aparición de los medios digitales, los niños están más expuestos que nunca a las acciones de marketing desarrolladas por las empresas. Ello, unido al cambio en su uso de los medios de comunicación -mayor navegación a través de Internet en lugar de ver la televisión, dada su preferencia por un medio interactivo-, implica su constante exposición a contenidos de lo más variado, algunos de los cuales pueden suponer riesgos para ellos, al realizarse sin la supervisión de un adulto.

En este sentido, es necesaria una actitud responsable por parte de las empresas. Muchos sitios web de alimentos dirigidos a los niños tratan de conseguir que estos pidan a sus padres la compra de los productos mostrados, ya que ofrecen no sólo información persuasiva acerca de los mismos, sino también juegos y otros contenidos, buscando cambios de actitud y de preferencias en sus hábitos alimentarios, que pueden desembocar en desórdenes en su salud física y mental, al mostrar a los niños los aspectos más placenteros del consumo de dichos productos sin poner de manifiesto sus aspectos negativos.

En el estudio realizado, se han analizado los sitios web de varias marcas de alimentos y bebidas dirigidos al público infantil, con el fin de analizar sus mensajes publicitarios y su posible influencia en los comportamientos del niño como consumidor.
\end{abstract}

PALABRAS CLAVE: Internet; infancia; alimentación; interactividad: publicidad online.

TITLE: Interactive communication strategies in food brands websites targeted to children

\section{ABSTRACT:}

This work is part of a wider investigation about advertising aimed at children and adolescents in interactive environments.

Many researchers are concerned about the effects that the content offered in the media can have on children. At present, and due to the emergence of digital media, children are more exposed than ever to the marketing activities undertaken by companies. This, coupled with the change in use of the media-most navigation through the Internet instead of watching TV because of its preference for an interactive media, involves the constant exposure to the most varied contents, some of which may pose risks to themselves, as this exposition is done without adult supervision.

1 La presente investigación ha sido realizada dentro del proyecto de investigación de la Universidad CEU San Pablo con referencia USP-PI-5/09, dirigido por María Sánchez Valle. Los autores desean manifestar su agradecimiento a la colaboración de Nielsen / Net-Rating Spain en el desarrollo del mismo. 
In this sense, we need a responsible attitude on the part of the companies that produce consumer goods. Many websites of food brands targeted to children are encouraging them to ask their parents to buy the products because they offer not only persuasive information about the products, but also games and other contents which can imply changes in attitude and preferences in their eating habits. This can also lead to effects such as higher rates of childhood obesity by showing children the most enjoyable aspects of the consumption of these products without highlighting their negative aspects.

In the study, we analyzed the websites of several brands of food and drinks aimed at children, in order to analyze their advertising messages and their possible influence on children behaviour as consumers.

KEY WORDS: Internet; children; food; interactivity; online advertising.

\section{Introducción}

En la última década se ha producido un incremento altamente significativo en el número de niños y adolescentes con problemas de sobrepeso en España. Un reciente estudio realizado entre niños y niñas de seis a trece años puso de manifiesto que el $13,9 \%$ de ellos tenían un peso por encima de lo recomendable para su edad, alcanzando incluso un grado considerable como obesidad ${ }^{2}$.

Los comportamientos alimentarios se educan y establecen durante la infancia, y se convierten en rutinas de por vida que marcan la salud del individuo. Es por ello que la obesidad y el sobrepeso infantil son problemas graves, que preocupan especialmente a las instituciones públicas y organismos responsables del sistema de salud $^{3}$, pues implican riesgos que pueden desembocar en enfermedades y dolencias crónicas graves para los adultos.

Hay diversos factores que pueden incidir en los comportamientos relativos a pautas nutricionales y la elección de los alimentos, pero entre ellos sin duda destaca la publicidad de los mismos. En este sentido, cada vez más se desarrollan acciones de publicidad y marketing online en sitios dirigidos a los niños, en los que se promueven hábitos alimentarios y se promocionan marcas y productos que claramente contravienen las pautas básicas para una alimentación saludable.

El objetivo de este trabajo es describir dichas estrategias, y analizar cómo algunas de las marcas del sector de la alimentación, que dirigen claramente sus productos al público infantil, desarrollan políticas poco éticas para fomentar el consumo de dichos alimentos a través de Internet.

2 DiRECCIÓN GENERAL DE SALUd PÚBLICA Y ALIMENTACIÓN (2007): «La alimentación en la Comunidad de Madrid», Documentos de salud pública, ${ }^{\circ} 115$.

$\mathrm{http} / / / \mathrm{www}$.madrid.org/cs/Satellite?blobcol=urldata\&blobheader=application\%2Fpdf\&blobheadername1=Conten t-disposition\&blobheadername $2=$ cadena\&blobheadervalue $1=$ filename $\% 3 \mathrm{DLa}+$ alimentacion+en+

la + Comunidad + de + Madrid + en+la $+w e b \% 2 C 0$.pdf\&blobheadervalue $2=$ language $\% 3$ Des $\% 26$ site $\% 3$ DPortalSalud \&blobkey=id\&blobtable=MungoBlobs\&blobwhere=1202746750589\&ssbinary=true. $W e b$ visitada el 2/09/2010.

3 La recientemente aprobada Ley de Seguridad Alimentaria y Nutrición restringe el uso de grasas trans, y abre la posibilidad de limitar la venta de «comida basura» en las escuelas

(http://www.la-moncloa.es/ConsejodeMinistros/Enlaces/030910-enlacealimentaria. Web visitada el 3/09/2010) 


\section{Infancia e Internet: usuarios y consumidores}

Los niños de hoy en día tienen un acceso a los medios y las tecnologías de la información mayores que nunca antes. Los menores pertenecen a la denominada como generación Einstein, generación ND (Nacido Digital), o generación C («Creatividad, Contenido, Compartir, Creatividad...») ${ }^{4}$. Son niños y jóvenes que han crecido en un mundo en el que la tecnología ha sido parte integral de sus vidas, y su lengua materna es el lenguaje digital de los dispositivos electrónicos como el ordenador o el móvil, y la conexión a Internet. En concreto, los nativos digitales tienen acceso a Internet desde su hogar en el $71 \%$ de los $\operatorname{casos}^{5}$, por lo que no es difícil que puedan acabar exponiéndose a las estrategias publicitarias de las empresas en la red. La relación entre los jóvenes y los entornos interactivos está suscitando un creciente interés tanto en el mundo académico como en el profesional, pues los jóvenes son un público atractivo desde un punto de vista comercial, y plantean un importante reto a sus educadores (padres, docentes, medios de comunicación incluso) sobre cómo acceder a ellos a pesar de la brecha digital existente entre una generación y otra ${ }^{6}$. En este sentido, destacan algunas investigaciones publicadas sobre el acceso de los menores a la red como el Estudio de Consumo de Medios en Europa de la Asociación Europea de Publicidad Interactiva (EIAA) ${ }^{7}$, o el Cuarto Observatorio de Tendencias Nokia ${ }^{8}$. Más centrado en el público infantil puede destacarse un amplio estudio del Eurobarómetro ${ }^{9}$, que muestra cómo los más pequeños acceden a la web, la mayoría desde sus casas, mediante conexiones de banda ancha, pero también desde la escuela, las casas de sus amigos, y en menor medida en bibliotecas y otros lugares públicos destinados al acceso a la red.

Estos patrones de acceso concuerdan también con un dato contrastado, y es que la mayoría acceden a Internet con uno de sus progenitores como iniciador, quien les explica el funcionamiento y utilidad de la red, pero en un breve plazo, los niños acceden por sí mismos y siguen perfeccionando sus niveles de conocimiento y uso

4 BúA, S. (2010): «La Generación C». Encuentro de Investigadores en Comunicación, Universidad CEU San Pablo, 22 de junio.

${ }^{5}$ BringuÉ, X. (2009): La Generación Interactiva en España. Niños y adolescentes ante las pantallas, Madrid, Fundación Telefónica.

6 Alonso, H.; BARTOLOMÉ, A. (2010): «Uso de las nuevas tecnologías en la docencia de publicidad y relaciones públicas», I Congreso Internacional Los Estudios de Comunicación en el EEES, Huesca, Universitat Abat Oliba, 8 de octubre.

7 EUROPEAN INTERACTIVE ADVERTISING ASSOCIATION (2010): «Internet se consolida como el medio más "consumido" por los españoles», http://www.eiaa.net/news/eiaa-articles-details.asp?id=218\&lang=5. Web visitada el 23/11/2009.

8 NoKIA (2010): Cuarto Observatorio de Tendencias Nokia: Los jóvenes, los móviles y la tecnología, disponible en http://www.youtube.com/watch?v=L4I09IeEZMI. Web consultada el 23/11/2009.

9 COMISIÓN EUROPEA (2007): Safer Internet for Children: Qualitative Studies in 29 European Countries Summary Report, http://ec.europa.eu/public_opinion/quali/ql_safer_internet_summary.pdf. Web visitada el 6/05/10. 
de Internet, bien por sí mismos, bien por la observación de sus amigos y compañeros, pero sin recurrir de nuevo a usuarios adultos. De hecho, el estudio pone de manifiesto cómo el aprendizaje del uso de la red se aproxima a las características del medio en sí: fácil, inmediato, y con una finalidad principalmente de entretenimiento o social, para la relación con su entorno, y en menor medida para fines educativos y didácticos.

La frecuencia e intensidad de uso de la red varía en función de la edad, pues los encuestados más jóvenes (menores de diez años) afirmaron conectarse a la red entre tres y cuatro veces a la semana, mientras que los usuarios de más edad, de doce a catorce años, reconocieron que se conectaban a diario, normalmente al final del día, ya a la vuelta de la escuela y, en teoría, una vez finalizados sus deberes. Así, los internautas de más edad pueden considerarse usuarios asiduos, que utilizan la red para divertirse con juegos en red, buscar información relevante y navegar por pura diversión.

Internet es un medio muy atractivo para las empresas que deseen dirigirse a un público eminentemente infantil por diversas causas ${ }^{10}$ :

- Es parte de la cultura juvenil. Esta generación de jóvenes está creciendo con Internet como un medio que forma parte de la rutina diaria de sus vidas.

- Los padres no son conscientes de la capacidad de las empresas para influir sobre sus hijos a través de los sitios web, por lo que no muestran preocupación por los contenidos a los que sus hijos acceden. De hecho, a menudo los menores acceden a la red solos, sin supervisión paterna.

- A diferencia de los medios tradicionales de radiodifusión, que tienen códigos deontológicos de autocontrol en materia de publicidad dirigida a los niños, no hay una regulación específica para Internet.

- Las sofisticadas tecnologías disponibles permiten a las empresas recoger con más facilidad un vasto volumen de información de los menores para la investigación de marketing, lo que a su vez facilita llegar más claramente a los niños mediante mensajes publicitarios personalizados.

- Mediante la creación de entornos interactivos atractivos, las empresas pueden construir lealtad de marca desde una edad temprana, máxime cuando en los websites se difuminan los límites entre la publicidad, el entretenimiento y la información, logrando que el niño no perciba que está siendo expuesto a un mensaje comercial. Así, los menores participan en videojuegos en los que la marca aparece claramente posicionada mediante elementos de identidad corporativa, acceden a páginas en las que sus personajes favoritos se asocian con el consumo del producto publicitado, obtienen ventajas al registrarse en el sitio, como participaciones en sorteos y concursos, y pueden descargarse

10 Media AWARENESS NETWORK (2010): How marketers target kids. http://www.mediaawareness.ca/english/parents/marketing/marketers_target_kids.cfm. Web consultada el 6/07/2010. 
recursos percibidos como un regalo por los niños, entre otras técnicas de marketing ${ }^{11}$.

\section{Estrategias de marketing interactivo de las marcas de productos de alimen- tación}

Las empresas fabricantes de productos dirigidos al público infantil se dirigen cada vez más a su target a través de Internet. Los propios anunciantes que reconocen que los niños no están capacitados para tomar decisiones de compra maduras hasta que no alcanzan una determinada edad, son los mismos que consideran apropiado dirigirse a ellos como consumidores antes incluso de cumplir los siete años ${ }^{12}$. Dado que los objetivos de las empresas se dirigen a ser reconocidas y consideradas por los niños en sus listas de preferencias, en un primer momento se persigue el reconocimiento de la marca, que el niño sea capaz de identificar logotipos, colores corporativos o packaging de los productos que a ellos se dirigen, para que posteriormente puedan solicitar a sus padres que se los compren.

En este sentido, el comportamiento de compra infantil está convenientemente estudiado, y los niños inician su andadura comercial acompañando a sus padres al supermercado y observando lo que les rodea. A partir de los dos años, ya son capaces no solo de acompañar a sus padres, sino de pedirles productos que les atraen, y establecer relaciones entre lo que ven en la tienda y lo que han visto anunciado en televisión. Con tres años, los niños están capacitados para reconocer marcas y productos dentro de las secciones del establecimiento, y ya colaboran con sus padres seleccionando diversos artículos bajo supervisión. Con cuatro años son capaces de realizar algunas selecciones de forma autónoma, y con cinco ya se desplazan solos por el establecimiento para elegir los productos de forma independiente según los requerimientos paternos ${ }^{13}$.

Para Young ${ }^{14}$, el niño evoluciona en su comprensión de los mensajes publicitarios desde la mera atención al mensaje, la distinción entre publicidad y programación, la interpretación del contenido e intencionalidad del anuncio, y la elaboración de defensas cognitivas ante este tipo de contenidos. Tanto Young como Bringué ${ }^{15}$

11 CAlvert, S.L. (2008): «Children as Consumers: Advertising and Marketing», Future Child, 18(1). Princeton, The Woodrow Wilson School of Public and International Affairs at Princeton University \& The Brookings Institution, 205-234.

12 DAHL, S., EAGLE, L., Y BAEZ, C. (2009): «Analyzing advergames: active diversions or actually deception. An exploratory study of online advergames content». Young Consumers, 10(1), 46.

13 BeDER, J. (1998): «The home visit, revisited», Families in Society: The Journal of Contemporary Human Services, 79, 103.

14 Martínez, S. (2005): «Los niños ante la publicidad televisiva», Comunicación y Hombre, 1, Madrid, Universidad Francisco de Vitoria, 199-209.

15 BRINGUÉ, X. (2000): «Publicidad infantil y estrategia persuasiva: un análisis de contenido», Zer, 10, Bilbao, Universidad del País Vasco, 107. 
consideran que la influencia de la publicidad sobre un sujeto, el niño, aún en su etapa de formación como persona, puede resumirse en tres campos de actuación:

- Modificación de sus actitudes y valores, a corto o largo plazo.

- Realización de peticiones a otras personas como los padres o los iguales.

- Influencia en las elecciones del niño y en su comportamiento de compra.

En relación con la idea de que los más pequeños no están plenamente capacitados para discernir sobre los mensajes comerciales que las marcas les hacen llegar, hay que considerar también las condiciones del acceso que los menores tienen a la red. Normalmente, los niños entran en sitios web diseñados con propósitos comerciales sin una supervisión directa de padres, profesores u otros adultos, y entran en contacto con juegos y otras acciones desarrolladas por las marcas, accediendo a abundante información sobre los productos, y dejando datos personales al alcance de los estrategas de marketing de las empresas ${ }^{16}$. Los sitios web incluyen todo tipo de acciones tales como concursos, juegos, secciones especiales sólo para usuarios registrados, etc. a través de las cuales las empresas obtienen abundante información, que puede ser luego empleada para crear estrategias personalizadas de marketing y ventas dirigidas directamente al niño ${ }^{17}$.

En este sentido, dentro de la estructura familiar, puede destacarse la creciente importancia de los niños y adolescentes en la toma de decisiones de compra. Ya no ejercen solamente papeles relevantes como iniciadores y/o expertos sino que participan activamente en las decisiones respecto de determinados productos, tanto de consumo inmediato (alimentación, por ejemplo) como duradero. Es lo que en la terminología anglosajona se denomina «skippies», school kids with income and purchasing power ${ }^{18}$.

De este modo, el colectivo infantil se convierte cada vez más en destinatario de la acción mercadotécnica de las compañías, que no sólo han comprobado la capacidad de los menores para influir en las decisiones de compra actuales, sino que además presentan un enorme potencial a largo plazo $^{19}$. Desde el primer enfoque, se es consciente de las posibilidades del mercado infantil dado su creciente capacidad económica, su vulnerabilidad y, sobre todo, su capacidad de influencia en los adultos que le rodean, tanto para compras de precio reducido como en otras de alto

16 COWBuRn, G. Y BoXer, A. (2007): «Magazines for children and young people and the links to Internet food marketing: a review of the extent and type of food advertising», Public Health Nutrition, 10 (10), 1025. http://www.ncbi.nlm.nih.gov/pubmed/17517153. Web consultada el 20/07/2010.

17 Story, M. Y FrenCH, S. (2004): «Food Advertising and Marketing Directed at Children and Adolescents in the US», International Journal of Behavioral Nutrition and Physical Activity, 13, http://www.ijbnpa.org/content/1/1/3. Web consultada el 07/10/2010.

18 ReISS, A. (2004): «Kleine Konsumenten: Analyse des Kaufverhaltens von Kindern im Grundschulalter», http://kirke.ub.uni-lueneburg.de/opus/volltexte/2004/239/pdf/mydiplomy.pdf, web consultada el 23 de mayo de 2007.

19 AlONSO, H.; GutiérREZ DE CABIEDES, L.; LÓPEZ, I. (2007): «Productos para adultos, publicidad para niños: los menores como prescriptores en los anuncios de automóviles, I Congreso Internacional La Televisión y la Infancia. Madrid, Universidad CEU San Pablo, 20 de octubre. 
coste, en las que moldean el consumo familiar en función de su propio criterio. Así, los niños juegan un papel importante en las decisiones de compra, especialmente en productos de consumo habitual como cereales, galletas, dulces, refrescos o yogures. Un estudio de Ward y Walkman ${ }^{20}$ revela que el porcentaje de concesión a las opiniones de los hijos en la compra de cereales para el desayuno alcanza el 8\%; en refrescos, el $46 \%$, y en productos para hacer bocadillos, el $63 \%$.

Pero sobre todo, los niños son vistos como potenciales consumidores adultos, que podrán aportar incrementos significativos en las ventas. Sobre ellos, las compañías pretenden suscitar una relación de lealtad que les acompañe a lo largo de toda su vida, al producto, a la marca, a un envase, a un eslogan... se trata de crear un vínculo emocional a largo plazo con el pequeño consumidor ${ }^{21}$. En este sentido, puede hablarse incluso de «confort brands», marcas que se utilizaron los padres durante la infancia y a las que se ligan vínculos emocionales positivos ${ }^{22}$.

\section{El Código PAOS}

Los impactos publicitarios recibidos por niños y adolescentes pueden influir claramente en sus preferencias de consumo. La mayor parte de los anuncios de productos de alimentación dirigidos al público infantil no hacen referencia a alimentos propios de las comidas principales del día ${ }^{23}$, sino precisamente a los aperitivos y dulces que los más pequeños prefieren tomar entre horas, y que pueden acabar generando diversos desórdenes en los patrones de alimentación de los más pequeños. De este modo, entre los riesgos asociados al consumo de dichos productos pueden citarse problemas de obesidad, enfermedades como anorexia o bulimia, prácticas deportivas inadecuadas, o problemas de autoestima vinculados a la imagen física personal entre otros comportamientos ${ }^{24}$. Diversos estudios han mostrado la correlación entre las acciones publicitarias desarrolladas por las empresas fabricantes de alimentos dirigidos al público más joven y el crecimiento en las tasas de

20 Prochile (2006): «Los hogares españoles», Segmentos Socioculturales, http://www.prochile.cl/newsletters/habitos_europa_2006/9_madrid_segmentos.php. Web consultada el 21/05/2007.

21 RAGNEDDA, M.; RuIU, M.L. (2010): «La violencia simbólica de la música en la publicidad destinada a la infancia», Pensar la Publicidad, 4 (1), 167-188

22 GÓMEZ, J.M.; BLANCO, J. (2005): «Los niños en la publicidad. Una propuesta de categorización de las representaciones sociales sobre la infancia en los anuncios televisivos», Zer, 19, Bilbao, Universidad del País Vasco, mayo, 53-76.

23 HARRISON, K.; MARSKE, A. L. (2005): «Nutritional content of foods advertised during the television programs children watch most», American Journal of Public Health, vol. 95 núm. 9, 1568-1574.

24 MORIARTY, C. M. Y HARRISON, K. (2008): «Television exposure and disordered eating among children: a longitudinal panel study», Journal of Communication, 58, 361-381. 
obesidad infanti1 ${ }^{25}$, así como entre dicha obesidad y el tiempo empleado por los menores en el consumo de medios de comunicación. En tercer lugar, cabe destacar la relación entre mayor presión publicitaria de las marcas y el deseo de compra suscitado en los menores ${ }^{26}$. Así, la adecuada alimentación infantil se convierte en un problema multifactorial que exige una respuesta coordinada de todos los organismos involucrados, que incluyen no sólo la industria alimentaria y el sector de la publicidad, sino también las autoridades de salud, padres, profesores, etc.

En el caso español, el Código de Autorregulación de la Publicidad de alimentos dirigida a menores ${ }^{27}$ (PAOS), forma parte del marco de la Estrategia NAOS lanzada por el Ministerio de Sanidad, cuyo objetivo es reducir la tendencia al sobrepeso y la obesidad, y sus consecuencias para la salud pública y sus repercusiones sociales, un objetivo compartido por las instituciones europeas y la OMS (Organización Mundial de la Salud). Dicho código tiene por objeto ayudar a las empresas gestionar sus mensajes dirigidos a menores de edad, especialmente los niños menores de 12 años, dada la gran influencia que puede tener sobre este público.

La entrada en vigor del Código PAOS implica, entre otras cuestiones, que cualquier persona, a título individual o a través de asociaciones de consumo, puede hacer una denuncia de forma gratuita a la Comisión de Autorregulación en relación a la publicidad de productos alimentarios. Del mismo modo, los firmantes han acordado que, antes de transmitir un anuncio, éste sea evaluado para comprobar que se adecue a las pautas que el código establece.

Así, respecto de la publicidad de alimentos y bebidas dirigida a menores, el Código PAOS establece como pautas básicas de actuación el principio de legalidad y de lealtad al afirmar que «la publicidad de alimentos y bebidas dirigida a menores se ajustará a las exigencias de la buena fe y los buenos usos mercantiles, cualquiera que sea su contenido, el medio de difusión o la forma que adopte». En este sentido, entre las normas principales que dicho código establece se encuentran las siguientes:

- Se adoptarán especiales cautelas en la realización y difusión de la publicidad de alimentos o bebidas dirigida a menores para garantizar que las imágenes y mensajes transmitidos no les induzcan a error acerca de las características del producto en cuestión.

- La presentación publicitaria de alimentos o bebidas no deberá inducir a error a los menores sobre los beneficios derivados del uso del producto, y espe-

25 TERLUTTER, R. et alia (2008): «The content and nature of food advertising in german children's tv programming», American Academy of Advertising Conference Proceedings, 202-206.

26 BRIDGES, E.; BRIESCH, R.A. (2006): «The nag factor and children's product categories», International Journal of Advertising, 25(2), 157-187.

27 El Código de Autorregulación de la Publicidad de alimentos dirigida a menores (PAOS) se firmó el 9 de junio de 2005, y contó con el apoyo de la Federación Española de Industrias de la Alimentación y Bebidas, FIAB. Actualmente, cuenta con 36 empresas adheridas, que suponen el 94,29\% de la inversión publicitaria en televisión de alimentos y bebidas dirigida a niños. Además, desde septiembre de 2009, las televisiones también están adheridas al Código. 
cialmente de aquéllos tales como la adquisición de fortaleza, estatus, popularidad, crecimiento, habilidad e inteligencia.

- Deberán adoptarse precauciones para no explotar la imaginación del menor, mediante el uso de la fantasía y la animación, de tal modo que dichos elementos creen expectativas inalcanzables o exploten la ingenuidad de los niños más pequeños.

- Cuando en un anuncio de alimentos o bebidas dirigido a menores sea necesario ofrecer cualquier información adicional, ésta deberá expresarse en un lenguaje comprensible para el público menor de edad y de forma clara, legible y destacada, considerando especialmente el tiempo de permanencia en pantalla de dicha información.

- La publicidad de alimentos o bebidas no debe hacer un llamamiento directo a los menores a la compra del producto, ni incitarles a que pidan o persuadan a sus padres o a otras personas para que compren los productos anunciados, o mostrar que aquellos adultos que sí compran el producto son mejores o más generosos.

- La publicidad de alimentos o bebidas en ningún caso explotará la especial confianza de los menores en sus padres, en profesores, o en otras personas, tales como profesionales de programas infantiles, o personajes (reales o ficticios) de películas o series de ficción. Esta medida será de especial aplicación en el caso de los productos con licencia ${ }^{28}$.

- El mensaje publicitario que incluya una promoción deberá diseñarse de tal forma que, además de transmitir el mensaje relativo al incentivo promocional, muestre claramente el producto anunciado. Las condiciones esenciales de las ofertas promocionales deben expresarse en la publicidad con sencillez y claridad, de forma que resulten fácilmente legibles y comprensibles para los menores.

- Las presentaciones comparativas deben ser mostradas de manera que los menores las entiendan con claridad.

- Los anuncios deben evitar las escenas, imágenes o mensajes que alienten el uso peligroso o inadecuado del producto anunciado.

En definitiva, se trata de evitar que las marcas puedan aprovechar la credulidad infantil en beneficio de sus productos, y por el contrario, fomentar una acción comercial basada en la transparencia, y que ayude a los menores a discernir entre las características de los diferentes productos basándose en la diferenciación real entre unos y otros, y no en características y de carácter más emotivo que racional, como los incentivos promocionales que se regalan, las prescripciones de personajes de referencia, o la presunción de supuestas ventajas para el consumidor de dichos productos.

28 La recién aprobada Ley de Seguridad Alimentaria y Nutrición incide en este sentido, al prohibir la prescripción de personajes famosos en la publicidad de alimentos. 


\section{Análisis de las estrategias de marketing en la red dirigidas al público infantil}

\subsection{Diseño metodológico del estudio}

Sobre el presente trabajo conviene reseñar en primer lugar que detalla las conclusiones de un estudio más amplio. El objetivo del mismo, como ya se ha comentado, es identificar las acciones que se están desarrollando en Internet por parte de las marcas de alimentación que incluyen en su cartera de productos algunos claramente dirigidos al público infantil.

Para realizar el estudio, se optó por la técnica del análisis de contenido, de modo que pudiesen constatarse de primera mano las estrategias desarrolladas por las marcas consideradas. En este sentido, se tomó como referente a las compañías que han suscrito el mencionado Código PAOS, con el fin de comprobar si en la red también estaban respetando los principios de éste. De cada una de dichas empresas se consideraron todos los sitios web disponibles, lo que incluía desde portales corporativos hasta microsites creados para acciones específicas. Así, se desecharon los sitios enfocados a la información corporativa, o aquéllos de marcas y productos dirigidos al público adulto. En definitiva, se eliminaron aquéllos que no presentaban interés para el público infantil, y que por tanto no debían ser tenidos en cuenta dado el objeto de estudio presente. Una vez seleccionadas las webs claramente orientadas al público infantil, se constató que existían veinticinco sitios, lo que se presentaba como una muestra suficientemente representativa. En ella se incluían tanto los sitios de algunas marcas, como microsites específicos creados para acciones promocionales concretas de producto.

Las veinticinco marcas analizadas en el estudio, son, por tanto: Central Lechera Asturiana (CAPSA), Danonino (Danone), Puleva Maxi, Batidos Puleva (Puleva), Hero Muesly (Hero), Kelloggs (varias marcas agrupadas en su site para niños), Royal (Kraft Foods), Doowap (La bella Easo), Yosport (Pascual), M\&Ms (Masterfoods), Nesquik, y dos microsites promocionales del grupo Nestlé; Phoskitos, Nocilla, Okay, Tosta Rica, Cola Cao, La Piara y Cuétara Flakes (Nutrexpa), Bizdonuts (Panrico), Cheetos, Matutano y Chipicao (Pepsico), y por último, el sitio de Calippo (Unilever). El estudio se realizó en la segunda quincena de julio de 2010. Por ello, es posible que algunos de los sites analizados hayan introducido variaciones, o que algunos microsites creados para acciones específicas no estén ya vigentes.

Conviene hacer notar, llegado este punto, que de los sitios analizados, hay dos globales de marca corporativa (los dos microsites de Nestlé), seis pertenecen a marcas de productos lácteos (Asturiana, Danonino, Yosport, Okay, y los dos sites de Puleva); cuatro, a marcas de cereales y galletas (Hero Muesly, Kelloggs, Tosta Rica y Cuétara Flakes) y dos son productos solubles a base de cacao (Cola Cao y Nesquik), tres categorías vinculadas a los desayunos y meriendas de los más pequeños; uno de helados, Calippo, otro de foie-gras (La Piara) y hasta nueve marcas (Royal, Doowap, M\&Ms, Phoskitos, Nocilla, Bizdonuts, Cheetos, Matutano y Chi- 
picao) pueden encuadrarse en categorías poco saludables como los postres semipreparados, los aperitivos o snacks y la bollería industrial.

Por otro lado, se trataba de definir las categorías a las que debía prestar atención el análisis. Existen numerosos estudios que analizan las acciones de marketing online de distintas compañías, enfocados tanto a otros sectores ${ }^{29}$, como a acciones concretas, por ejemplo, la venta online $e^{30}$ o la publicidad en otros sites distintos del corporativo $^{31}$, o bien para mercados geográficos distintos del español. En este caso, la bibliografía consultada fue de gran utilidad, pues permitió comparar la situación de nuestro país frente a otros con legislaciones más estrictas en lo que a Internet y público infantil se refiere. Así, se tomó como base un reciente estudio del mercado australiano $^{32}$, y se consideraron las categorías propuestas en él, añadiendo, suprimiendo y modificando algunas, en función de las particularidades de las acciones desarrolladas por las empresas españolas.

Así, los aspectos analizados para cada una de ellas son los siguientes: identificadores de marca, juegos, promociones, personajes populares, enlaces, descargas, beneficios del producto, cuentas y registro, educación y protección del menor.

En la categoría de identificadores de marca se trataba de analizar los elementos de branding empleados por las empresas. Así, «Logos» hace referencia a las empresas que incluyen los logotipos y otros elementos de identidad corporativa en sus sitios web. "Packaging" se refiere a la inclusión de la imagen del producto tal y como se comercializa en el punto de venta, incluso con mención de los diversos formatos disponibles.

Juegos: hace referencia a los juegos y pasatiempos que cada marca incluye en sus sites. Distinguimos por un lado «Advergaming/general», las marcas que incluyen juegos en sus propios sites como una oferta de entretenimiento para los más pequeños, frente a las que van más allá, y ofrecen juegos en los que además el producto se integra en la acción («Advergaming+marca»).

Promociones: acciones promocionales desarrolladas por las empresas tanto específicas del sitio como integrando las desarrolladas en el punto de venta. Así, «Concursos/premios» hace referencia a las acciones pensadas exclusivamente para la web, y «Promociones offline» se refiere a las empresas que informan a través de su website de promociones a las que puede acceder el consumidor comprando el producto en un punto de venta offline (supermercados, etc.). Dichas promociones

29 SINHA, R. et alia (2001): «Content or graphics? An empirical analysis of criteria for award-winning websites», Proceedings of the 7th Conference on Human Factors \& the web, http://www.sims.berkeley.edu/ sinha/papers/sinha_hfw01.pdf. Web visitada el 20/06/2010

30 VAN DER MERWE, R.; BEKKER, J. (2003): «A framework and methodology for evaluating ecommerce websites», Internet research: Electronic Networking Policy, 5 (13), 333-334.

31 SÁNCHEZ, M.; Frutos, B. (2010): «La investigación de la publicidad interactiva dirigida a niños y adolescentes». Comunicación y Desarrollo en la era digital, http://www.aeic2010malaga.org/upload/ok/206.pdf. Web visitada el 8/09/2010

32 Kelly, B. et alia (2008): «Internet food marketing on popular children's websites and food products websites in Australia», Public Health Nutrition, 11 (11), 1180-1187. 
invitan al niño a consumir de forma creciente el producto, para así poder acceder de forma directa al incentivo en cuestión, o bien incrementar sus posibilidades de lograrlo en el caso de los sorteos y concursos. La investigación sobre «Fundraising» trata de detectar si alguna empresa trataba de promover una compra mayor o más frecuente del producto (compartiendo así objetivo con las promociones de ventas) al poner de manifiesto la acción social desarrollada por las empresas. Por último, «Muestras de producto», con el objetivo de identificar si a través de la web era posible conseguir el envío de muestras del producto.

Personajes populares: en este apartado se trata de conocer si las marcas apelan al valor que para los niños puede tener la prescripción de personajes conocidos. Hay que tener en cuenta que ésta es una de las cuestiones más polémicas, al ser una clara recomendación del Código PAOS la evitación de estas acciones para no influir en los mecanismos de comportamiento de los menores. Así, se han analizado cuatro categorías, «Propios», para aquellas marcas (52\%) que incluyen en la web las mascotas o personajes propios creados para vender el producto, frente a las que acuden al valor de personajes conocidos para el público infantil, tanto reales como de ficción, y que se han encuadrado en las categorías «Deportistas» ${ }^{33}$, «Famosos»y «Dibujos».

Enlaces: esta sección hace referencia a los links que pueden encontrarse desde los sitios analizados a otros que puedan ser atractivos para el target al que se dirigen, mejorando la percepción de marca. Así, «Enlaces - alimentación» se relaciona con páginas web vinculadas al mundo de la nutrición, $\mathrm{y}$ «Enlaces - no alimentación», con sitios informativos sobre temas tales como deportes, música, juegos, etc. «Enlaces - marcas» se refiere a las referencias (textuales o fotográficas) a otros productos de la misma empresa fabricante. Por último, se consignó en la categoría «Anuncios» a las marcas que incluían enlaces a los anuncios de televisión de la marca.

Descargas: descargas disponibles de diversos tipos de contenidos interesantes para el target infantil, tales como fondos de escritorio, salvapantallas, politonos, emoticonos u otros.

Beneficios del producto: en esta sección se recoge la información que los sites incluyen sobre los beneficios que el producto puede aportar con su consumo. Así, «Beneficios de la marca» se refiere a los mensajes positivos sobre la marca y sus ventajas. «Información nutricional» hace mención a las marcas que incluyen datos sobre ingredientes y valor alimentario de cada uno de sus productos, de forma más objetiva, frente a las que incorporan dicha información («Información / lemas») presentándola en relación con las ventajas nutricionales de sus productos. También en esta línea se analizaron las categorías «Información de salud», si las empresas incluían consejos sobre hábitos saludables, al margen de la alimentación (por ejemplo, la promoción del deporte o los hábitos higiénicos adecuados) y «Lemas

33 Se decidió separar la categoría «Deportistas» de otros famosos como puedan ser cantantes, actores, etc., dado el importante valor de prescripción que pueden tener no sólo como personajes conocidos y admirados por los más pequeños, sino además porque su éxito profesional viene dado en parte por unas condiciones físicas concretas que el niño podría asociar con el consumo del producto anunciado. 
sobre salud», cuando se trataba de incluir ventajas para la salud derivadas del consumo de sus productos, y no sólo referidas al aporte nutricional/calórico, sino también a la protección frente al colesterol, el crecimiento, etc.

Cuentas y registro: esta sección hace referencia a la relación que se establece entre el usuario del website y la marca. En este sentido, se analizan aspectos como la posibilidad de ser un usuario registrado («Registro en el sitio»), e incluso aquellas compañías que, yendo más allá, ofrecen beneficios específicos sólo para usuarios registrados, tales como el acceso a contenidos premium o la participación en juegos y concursos. «Marketing viral» se refiere a las marcas que permiten enviar la página a un amigo, publicarla mediante RSS, o indicar preferencias y gustos en redes sociales desde el propio sitio. «Comunidades» hace referencia a los grupos que pueden crearse en torno a la marca, con más o menos posibilidades de contacto entre usuarios, a través de páginas de admiradores en redes sociales externas o propias de la empresa, y por último, «Tienda online», en el caso de las compañías que ofrecen la posibilidad de adquirir sus productos en el propio sitio de la marca.

Educación: Se refiere a la inclusión de contenidos no comerciales y de carácter educativo para los más pequeños.

Protección de datos: trata cuestiones fundamentales como la seguridad y protección de los menores, respecto de su privacidad y los contenidos comerciales a los que están expuestos en las redes. Así, se ha analizado si los sitios incluyen la necesaria «Información legal», si se advierte a los menores de forma comprensible sobre el uso de «Cookies», o si se informa a los menores sobre las cuestiones relativas al necesario «Consentimiento paterno».

\subsection{Resultados del estudio}

De acuerdo con las categorías citadas para el análisis, se obtuvieron los siguientes resultados:

Identificadores de marca: Todas las compañías analizadas incorporan el logo de las marcas específicas de alimentos infantiles en los sites, e incluso algunas, como Nestlé, incluyen el logotipo de la marca global. Respecto al packaging, la mayor parte de las marcas también incluyen esta referencia, con la excepción de los microsites especiales de Nestlé -que no hacen referencia a ningún producto concreto de la empresa- y el sitio de Calippo. Además, la mitad de los sites analizados incorporan toda la información referida a variedades y formatos comercializados. Se trata de que el niño pueda tener en mente e identificar los productos de las marcas contactadas online.

Juegos: en relación con los juegos y pasatiempos que cada marca incluye en sus sites, conviene destacar que el sesenta por ciento de las marcas incorporan juegos en sus sitios, pero además en algunos casos el producto se integra en la acción («Advergaming+marca»), como en el caso de M\&Ms, Nocilla, Bizdonuts, Calippo o La Piara. En este último caso, por ejemplo, se trata de un juego de plataformas en 
el que el personaje gana energía cada vez que atrapa uno de los populares envases de tapa negra de esta marca. De forma indirecta, pues no hay menciones específicas, pero se transmite al niño la idea de que este alimento puede hacerle más fuerte, contraviniendo las recomendaciones del código PAOS al respecto.

Promociones: La mitad de las empresas (48\%) incluyen concursos y juegos que en muchos casos permiten figurar en un ranking de ganadores o incluso ganar premios y participar en sorteos -como posteriormente se explica, esta opción obliga en muchos casos al registro del usuario mediante una dirección de email-. Respecto de las promociones ofrecidas en el punto de venta offline (supermercados, etc.), en ocasiones, simplemente se informa de la promoción, como en el caso de las marcas del grupo Nutrexpa (Cola Cao, Nocilla, Phoskitos), que informan al niño sobre los regalos que puede ganar si compra el producto, mientras que otras marcas como Danone, Batidos Puleva o Royal invitan a visitar la web para conseguir determinados regalos promocionales, dado que es necesario acceder a ella para introducir el código del lote del producto para saber si se ha ganado el premio en cuestión. Para las marcas, es muy interesante, pues se emite una comunicación mucho más coherente desde todos los canales, y además ofrecen la ventaja al consumidor de poder acceder por el canal que le resulte más ventajoso en cada momento (por ejemplo, elegir entre enviar un código de barras por correo postal o introducir el código del producto en una página del site). Otros dos aspectos que consideramos interesantes para la investigación eran el de «Fundraising», si las marcas informaban en sus sitios sobre sus acciones de Responsabilidad Social Corporativa, o si animaban a su público a participar en ellas, y «Muestras de producto», si a través de la web era posible conseguir el envío de muestras del producto, pero no se ha encontrado ninguna referencia en los sitios analizados.

Personajes populares: hasta el 52\% de las marcas incluyen en la web las mascotas o personajes propios creados para vender el producto, como los famosos Tony, Cornelius o Coco de Kelloggs, u otros de más reciente creación como Quicky de Nesquik, o Danonino de Danone. También otras marcas (24\%) apelan a personajes de dibujos muy conocidos por el público infantil como Shrek (Royal), Bob Esponja (Nocilla, Tosta Rica) o Hello Kitty (Okey, Cheetos). Asimismo, Central Lechera Asturiana utiliza como reclamo a la selección española de baloncesto, y Nestlé juega con la figura de Papá Noel. No se han encontrado en el momento del análisis otros reclamos tales como cantantes, actores, etc. En todo caso, los dibujos animados son el reclamo más usado por las marcas para que los sitios sean atractivos para los niños. Pueden ser mascotas propias de las marcas, o bien dibujos famosos del cine y la televisión, pero acompañan al niño en su proceso de navegación online y les suponen una asociación positiva con la marca.

Enlaces: esta sección hace referencia a los links que pueden encontrarse desde los sitios analizados a otros que puedan ser atractivos para el target al que se dirigen. Así, esperábamos que los sitios enlazasen con webs informativas (información general, deportes, música, nutrición u otros temas de interés para el público infantil), pero res- 
pecto de los sites analizados sólo cabe reseñar que el 36\% de ellos permiten entrar en otros websites de alimentación («enlaces- alimentación») de marcas del grupo. Nocilla, Tosta Rica y Cola Cao, todas marcas del grupo Nutrexpa, enlazan con sites dedicados exclusivamente a juegos («enlaces- no alimentación»). También cabe destacar lo que hemos denominado «Enlaces - marcas», las marcas $(20 \%)$ que incluyen referencias (textuales o fotográficas) a otros productos del grupo. Así, en el site de Nesquik se nos invita a hacer una receta con masa de hojaldre «La Cocinera», o Tosta Rica menciona la composición de un desayuno equilibrado, en el que no puede faltar Cola Cao. Asimismo, la mitad de las empresas (52\%) permiten acceder a los vídeos de los spots que actualmente se exhiben en televisión, algunas desde su propio site, otras mediante un enlace al sitio de You Tube.

Descargas: descargas disponibles de diversos tipos de contenidos interesantes para el target infantil. Dichas descargas son percibidas por los niños como un premio o regalo por acceder al sitio, y además permiten seguir ejerciendo contacto gráfico con ellos una vez que han salido del site. Si tenemos en consideración que en muchos casos es necesario registrarse para lograr la descarga, se entiende su gran valor promocional. Entre las descargas más populares se encuentran los fondos de escritorio (36\%), tonos para el móvil (16\%), salvapantallas (12\%), páginas para colorear (12\%), emoticonos (12\%), códigos bidi (8\%) y otros elementos como $(32 \%)$ tarjetas de invitación, maquetas, recortables, libros de pasatiempos, recetas fáciles, fotografías, etc.

Beneficios del producto: en esta sección se recoge la información que los sites incluyen sobre los beneficios que el producto puede aportar con su consumo. El $36 \%$ por ciento de las marcas incluyen apelaciones claras a los beneficios y ventajas que puede aportar el producto, respecto de valores más emocionales que racionales como la autenticidad (La Piara) o la originalidad (M\&Ms). Algunas de las marcas analizadas (40\%) incorporan la información nutricional, sobre ingredientes y valor alimentario de cada uno de sus productos, y un porcentaje similar incluye también lemas sobre nutrición, mensajes comerciales sobre las ventajas nutricionales de sus productos. En algunos casos, el bajo contenido en grasas o azúcar frente a otros productos similares, o bien la alta aportación energética, que permite combinar el producto con otros más saludables como yogur, leche o fruta para conseguir un desayuno o merienda equilibrados. En relación con la información nutricional, también puede hablarse de la información sobre hábitos saludables («Información de salud»), en el caso de las empresas que ofrecen información útil como la composición de un desayuno adecuado, o las ventajas del consumo de los ingredientes contenidos en sus productos. Así, uno de cada tres sitios analizados incorporaba «Lemas sobre salud», ventajas para la salud derivadas del consumo de sus productos, y no sólo referidas al aporte nutricional/calórico, sino también a la protección frente al colesterol, el crecimiento, etc. Por ejemplo, Royal permite rellenar un test en el que las respuestas correctas hacen referencia a las ventajas de sus productos. 
Cuentas y registro: El 56\% de los sites analizados ofrecen a los niños la posibilidad de registrarse mediante una dirección de email, introduciendo asimismo otros datos de gran interés para la investigación de mercados, puesto que se les solicita la edad, sexo, lugar de residencia, etc. En el sitio de Phoskitos incluso se invita al niño a decir cuáles son sus webs favoritas. El hecho del registro implica ventajas para los usuarios, como la participación en sorteos en el caso de Chipicao o Nocilla, e incluso se hace imprescindible en algunos sites para poder acceder a todos sus contenidos, por ejemplo para poder hacer uso de los juegos en el caso de Nestlé o Bizdonuts.

Una de cada tres marcas aprovecha las posibilidades de su site para el marketing viral, pues a través del sitio permiten enviar la página a un amigo, publicarla mediante RSS, o indicar su interés por un determinado contenido en algunas redes sociales como Facebook o Tuenti. También los sitios quieren aprovechar sus posibilidades para crear comunidades de usuarios («Comunidades»), en el propio site, en sus páginas de fans en redes sociales, o a través de herramientas más complejas como el caso de las comunidades de Habbo Hotel de Calippo y Yosport.

Por último, hay que indicar que dos empresas, Nocilla y Nesquik, disponen de tiendas online en las que el niño puede comprar productos con la identidad corporativa de su producto favorito: colchonetas, tostadoras, camisetas...La compra no se efectúa online mediante tarjeta de crédito, sino contra reembolso del envío de un cupón cumplimentado por correo ordinario, por lo que el control paterno desaparece en el caso de los niños de más edad, que pueden tener acceso al correo postal.

Educación: Puleva Maxi es la única marca que en su site incluye otros contenidos educativos para los niños, como información sobre cuidado del medioambiente, el crecimiento y sus etapas, etc. En este sentido, parece que las marcas consideran que los niños acceden a Internet con la finalidad del entretenimiento, no la de informarse o aprender.

Protección de datos: hace referencia a la seguridad y protección de los menores, respecto de su privacidad y los contenidos comerciales a los que están expuestos en las redes. En este sentido, cabe destacar que aunque la mayoría de los sites $(76 \%)$ incluye la información o aviso legal sobre condiciones de uso del sitio, muchos de ellos, como los de Cuétara y Okey, sólo la ofrecen en usos específicos como en el registro o si el usuario quiere reenviar la página web. Sólo las marcas del grupo Puleva incluyen una referencia clara fuera del aviso legal sobre el uso de «cookies» en el sitio, y Danone informa en la página de inicio del sitio a los padres de la posibilidad de dejar a los niños navegar solos en la página, justificando dicha postura al afirmar que en el site pueden encontrar sólo juegos y contenidos adaptados al público infantil. Asimismo, apenas una de cada cinco empresas indica claramente que es necesario el consentimiento paterno para el acceso a los contenidos, y obliga a introducir el email o NIF del padre, madre o tutor del niño para permitirle el acceso. En cuanto a esta cuestión, la facilidad del registro y la imposibilidad de contrastar los datos vertidos por los menores al cumplimentar dichos formula- 
rios, especialmente en lo concerniente a la edad, puede contravenir no sólo la lógica ética publicitaria, sino también la legislación referida a la protección de datos ${ }^{34}$.

\section{Conclusiones}

Los menores acceden cada vez en mayor medida a Internet, como medio de información, comunicación y entretenimiento, dado el sentimiento positivo que para ellos supone la interactividad de la red, frente a medios de comunicación unidireccional como la televisión. El principal problema derivado de esta práctica radica en la habitual ausencia de control paterno durante el acceso, lo que convierte a la red, por éste y otros motivos, en el medio idóneo para que las marcas puedan contactar de forma más directa con los niños y jóvenes. El creciente poder adquisitivo y de decisión dentro de la familia de los menores, unido a su potencial como futuros consumidores adultos, hace de ellos un objetivo fundamental de las acciones comerciales de las empresas.

La correlación entre consumo de medios de comunicación, presión publicitaria de las empresas fabricantes de productos de alimentación dirigidos al público infantil, y el incremento de la obesidad y otras patologías entre los menores, ha hecho saltar las alarmas de un problema que implica a empresas, autoridades, padres, docentes, medios de comunicación, etc. La falta de legislación y de control interno a través de la adecuada deontología profesional ha convertido a Internet en el canal ideal para la realización de acciones de marketing que se alejan bastante de los códigos éticos que las mismas compañías propugnan para otros medios. No se trata de prohibir la comercialización y comunicación sobre productos dirigidos a los más pequeños, pero sí de adecuar las acciones desarrolladas por las empresas a la lógica ética requerida cuando se trata de públicos tan sensibles y débiles frente a los estímulos comerciales.

Entre las acciones desarrolladas por las empresas que pueden ser consideradas poco éticas pueden citarse los juegos y concursos ofrecidos en la web en los que el producto interviene para comunicar de forma indirecta los supuestos beneficios que ofrecerá a quien lo consuma; el desarrollo de promociones que incitan al niño a pedir la compra de mayor cantidad y con mayor frecuencia de un determinado producto, para conseguir el incentivo promocional deseado; la prescripción a través del reclamo de personajes ficticios o reales que atraen al público infantil; la presentación de los beneficios del producto incidiendo en aspectos emocionales y minimizando los elementos perjudiciales de su composición, y la vinculación con los usuarios de los sitios a través de opciones de registro, en ocasiones imprescindibles para acceder a determinados contenidos del sitio, en las que se piden determinados

34 EFE (2010): «Facebook eleva a 14 años la edad mínima para entrar en su red española», http://www.elmundo.es/elmundo/2010/02/18/navegante/1266493878.html, web consultada el 12/11/2010. 
datos personales sin informar claramente del uso de la información y las condiciones legales para el acceso de los menores al site.

Dichas acciones contravienen las recomendaciones asumidas por las empresas a través del Código PAOS, e invitan a la reflexión a todos los actores implicados, para lograr una comunicación comercial ética, que ayude a dar a conocer los beneficios y ventajas de las marcas sin entrar en colisión con los principios éticos requeridos en la comunicación con los menores.

\section{Referencias bibliográficas}

Alonso, H.; BARTOlomé, A. (2010): «Uso de las nuevas tecnologías en la docencia de publicidad y relaciones públicas», I Congreso Internacional Los Estudios de Comunicación en el EEES, Huesca, Universitat Abat Oliba, 8 de octubre.

Alonso, H.; GutiÉRREZ DE CABIEDES, L.; LóPEZ, I. (2007): «Productos para adultos, publicidad para niños: los menores como prescriptores en los anuncios de automóviles», $I$ Congreso Internacional La Televisión y la Infancia, Madrid, Universidad CEU San Pablo, 20 de octubre.

BEDER, J. (1998): «The home visit, revisited», Families in Society: The Journal of Contemporary Human Services, 79.

BRIDGES, E. Y BRIESCH, R.A. (2006): «The nag factor and children's product categories", International Journal of Advertising, 25(2), 157-187.

BRINGUÉ, X. (2000): «Publicidad infantil y estrategia persuasiva: un análisis de contenido», Zer, 10, Bilbao, Universidad del País Vasco, 107.

BRINGUÉ, X. (2009): La Generación Interactiva en España. Niños y adolescentes ante las pantallas, Madrid, Fundación Telefónica.

BÚA, S. (2010): «La Generación C», Encuentro de Investigadores en Comunicación, Universidad CEU San Pablo, 22 de junio.

CALVERT, S.L. (2008): «Children as Consumers: Advertising and Marketing», Future Child, 18(1), Princeton, The Woodrow Wilson School of Public and International Affairs at Princeton University \& The Brookings Institution, 205-234.

COMISIÓN EUROPEA (2007): Safer Internet for Children: Qualitative Studies in 29 European Countries Summary Report, http://ec.europa.eu/public_opinion/quali/q1_safer_internet_summary.pdf. Web visitada el 6/05/10, Contemporary Human Services, $\overline{79}, 103$.

COWBURN, G.; BOXER, A. (2007): «Magazines for children and young people and the links to Internet food marketing: a review of the extent and type of food advertising», Public Health Nutrition, 10 (10), 1025, http://www.ncbi.nlm.nih.gov/pubmed/17517153. Web consultada el 20/07/2010.

DAHL, S.; EAGLE, L.; BAEZ, C. (2009): «Analyzing advergames: active diversions or actually deception. An exploratory study of online advergames content», Young Consumers, 10(1), 46.

DIRECCIÓN GENERAL DE SALUD PÚBLICA Y ALIMENTACIÓN (2007): «La alimentación en la Comunidad de Madrid», Documentos de salud pública, 115. 
http://www.madrid.org/cs/Satellite?blobcol=urldata\&blobheader=application\%2Fpdf\&b lobheadername $1=$ Content-disposition \&blobheadername $2=$ cadena\&blobheadervalue 1 $=$ filename $\% 3 \mathrm{DLa}+$ alimentacion $+\mathrm{en}+\mathrm{la}+\mathrm{Comunidad}+\mathrm{de}+$ Madrid + en $+\mathrm{la}+w e b \% 2 \mathrm{C} 0 . \mathrm{pdf}$ \&blobheadervalue2=language\%3Des\%26site\%3DPortalSalud\&blobkey=id\&blobtable= MungoBlobs\&blobwhere $=1202746750589 \&$ ssbinary $=$ true. $W e b$ visitada el 2/09/2010.

EFE (2010): «Facebook eleva a 14 años la edad mínima para entrar en su red española», http://www.elmundo.es/elmundo/2010/02/18/navegante/1266493878.html, web consultada el 12/11/2010.

EUROPEAN INTERACTIVE ADVERTISING ASSOCIATION (2010): «Internet se consolida como el medio más "consumido" por los españoles», http://www.eiaa.net/news/eiaa-articlesdetails.asp?id=218\&lang=5. Web visitada el 23/11/2009.

GóMEZ, J.M.; BLANCO, J. (2005): «Los niños en la publicidad. Una propuesta de categorización de las representaciones sociales sobre la infancia en los anuncios televisivos», Zer, 19. Bilbao, Universidad del País Vasco, mayo, 53-76.

HARRISON, K.; MARSKE, A. L. (2005): «Nutritional content of foods advertised during the television programs children watch most», American Journal of Public Health, 95 (9), 1568-1574.

KELLY, B. et alia (2008): «Internet food marketing on popular children's websites and food products websites in Australia», Public Health Nutrition, 11(11), 1180-1187.

MARTíneZ, S. (2005): «Los niños ante la publicidad televisiva», Comunicación y Hombre, 1, Madrid, Universidad Francisco de Vitoria, 199-209.

MEDIA AWARENESS NETWORK (2010): How marketers target kids, http://www.mediaawareness.ca/english/parents/marketing/marketers_target_kids.cfm. Web consultada el 6/07/2010.

MORIARTY, C. M.; HARRISON, K. (2008): «Television exposure and disordered eating among children: a longitudinal panel study», Journal of Communication, 58, 361-381

NOKIA (2010): Cuarto Observatorio de Tendencias Nokia: Los jóvenes, los móviles y la tecnología, disponible en http://www.youtube.com/watch?v=L4I09IeEZMI. Web consultada el 23/11/2009.

ProchILE (2006): «Los hogares españoles», Segmentos Socioculturales, http://www.prochile.cl/newsletters/habitos_europa_2006/9_madrid_segmentos.php. web consultada el 21/05/2007.

RAGNEDDA, M.; RuIU, M.L. (2010): «La violencia simbólica de la música en la publicidad destinada a la infancia», Pensar la Publicidad, 4(1), 167-188

REISS, A. (2004): «Kleine Konsumenten: Analyse des Kaufverhaltens von Kindern im Grundschulalter", http://kirke.ub.uni-lueneburg.de/opus/volltexte/2004/239/pdf/mydiplomy.pdf. Web consultada el 23 de mayo de 2007.

SÁNCHEZ, M.; FRUTOS, B. (2010): «La investigación de la publicidad interactiva dirigida a niños y adolescentes», Comunicación y Desarrollo en la era digital, http://www.aeic2010malaga.org/upload/ok/206.pdf. Web visitada el 8/09/2010

SINHA, R. et alia (2001): «Content or graphics? An empirical analysis of criteria for awardwinning websites», Proceedings of the 7th Conference on Human Factors \& the web, http://www.sims.berkeley.edu/ sinha/papers/sinha_hfw01.pdf. Web visitada el 20/06/2010. 
StORY, M.; FRENCH, S. (2004): «Food Advertising and Marketing Directed at Children and Adolescents in the US», International Journal of Behavioral Nutrition and Physical Activity, 13, http://www.ijbnpa.org/content/1/1/3. Web consultada el 07/10/2010.

TERLUTTER, R. et alia (2008): «The content and nature of food advertising in german children's tv programming», American Academy of Advertising Conference Proceedings, 202-206.

VAN DER MERWE, R.; BEKKER, J. (2003): «A framework and methodology for evaluating ecommerce websites», Internet research: Electronic Networking Policy, 5(13), 333-334.

Recibido: 31 de noviembre de 2010

Aceptado: 26 de septiembre de 2011 